\title{
The Impact of COVID-19 on Diabetic Retinopathy Monitoring and Treatment
}

\author{
Ishrat Ahmed ${ }^{1} \cdot$ T. Y. Alvin Liu ${ }^{1}$ \\ Accepted: 9 July 2021 / Published online: 8 September 2021 \\ (c) The Author(s), under exclusive licence to Springer Science+Business Media, LLC, part of Springer Nature 2021
}

\begin{abstract}
Purpose of Review Diabetic retinopathy (DR) is one of the leading causes of vision loss worldwide. Although screening and early treatment guidelines for DR have significantly reduced the disease burden, restrictions related to the COVID-19 pandemic have changed real-world practice patterns in the management of DR. This review summarizes evolving guidelines and outcomes of the treatment of DR in the setting of the pandemic.

Recent Findings Intravitreal injections for DR have decreased significantly globally during the pandemic, ranging from approximately 30 to nearly $100 \%$ reduction, compared to corresponding timepoints in 2019. Most studies on functional outcomes show a decrease in visual acuity on delayed follow-up.

Summary Changing practice patterns in the management of DR has led to fewer intravitreal injections and overall reduction in visual acuity on follow-up. As COVID variants emerge, it will be necessary to continue evaluating practice guidelines.
\end{abstract}

Keywords COVID-19 $\cdot$ Diabetic retinopathy $\cdot$ Diabetic macular edema $\cdot$ Intravitreal injections $\cdot$ Visual acuity $\cdot$ Practice patterns

\section{Introduction}

The coronavirus disease 2019 (COVID-19) pandemic has resulted in significant morbidity and mortality globally with $133,552,774$ confirmed cases and 2,894,295 deaths as of April 2021 [1]. COVID-19 can present with a myriad of systemic manifestations, including severe acute respiratory syndrome coronavirus 2 (SARS-CoV-2), that are mediated through the angiotensin-converting enzyme 2 (ACE2) receptor [2]. On a public health level, the impact of this pandemic, particularly on the management and outcomes of chronic diseases such as diabetic retinopathy (DR), is beginning to manifest. DR is diagnosed in approximately one third of patients with diabetes mellitus and is one of

This article is part of the Topical collection on Microvascular Complications-Retinopathy

T. Y. Alvin Liu

tliu25@jhmi.edu

Ishrat Ahmed

iahmed2@jhmi.edu

1 Wilmer Eye Institute, Johns Hopkins University, $600 \mathrm{~N}$. Wolfe Street, Maumenee 726, Baltimore, MD 21287, USA the most common causes of vision impairment worldwide $[3,4]$. Furthermore, despite guidelines for annual screening exams, approximately one-third of patients with diabetes do not adhere to these screening recommendations [5]. Regular screening and early treatment for DR have been shown to prevent severe vision loss and are cost effective $[6,7]$.

COVID-19 infection control guidelines, such as social distancing and appropriate use of personal protective equipment (PPE), as well as patient concerns, have affected outpatient appointment attendance during the pandemic $[8,9]$. Analysis of over 2 million patient encounters in the USA through the National Patient and Procedure Volume Tracker (Strata Decision Technology, L.L.C., Chicago, IL) showed a decline in clinic visits across all specialties, including an $81 \%$ decline in ophthalmology clinic visits in March and April 2020 compared to the same period in 2019 [10]. Retina clinics experienced about a $71 \%$ volume loss [10]. Unlike other specialties, the transition to tele-ophthalmology visits was limited, as ophthalmological care is heavily dependent on specialized imaging equipment that is not readily available outside of ophthalmologists' offices [11]. DR screening was also frequently postponed during the pandemic, particularly in areas with high COVID-19 community transmission [12]. For example, at the Wilmer Eye Institute, diabetic eye 
exams decreased from 1,145 visits in the 6 weeks prior to pandemic-related clinical practice changes to just 59 in the first 6 weeks after these changes were implemented [13].

This review will focus on the modified guidelines for DR monitoring and treatment as recommended by the American Academy of Ophthalmology and Vision Academy Steering Committee, patient perceptions towards ophthalmology clinic visits during the pandemic, change in the frequency of intravitreal injections, and resultant changes in outcomes such as visual acuity.

\section{Diabetic Retinopathy Treatment Guidelines During the Pandemic}

Elderly patients and patients with systemic co-morbidities, such as patients with diabetes requiring intravitreal injections, are at increased risk of developing severe illness from COVID-19 infection [14]. As such, on March 18, 2020, at the onset of the COVID-19 pandemic in the USA, the American Academy of Ophthalmology recommended that "all ophthalmologists cease providing any treatment other than urgent or emergent care immediately." Since then, the AAO has provided updated recommendations to reduce COVID19 exposure risks during outpatient clinic visits and elective surgeries. These recommendations include pre-clinic screening for COVID-19 symptoms, social distancing in waiting rooms, frequent disinfection, slit-lamp barriers and breath shields, and facial coverings for patients and caregivers during clinic visits, as well as COVID-19 RT-PCR testing and appropriate PPE for surgery [15]. Implementation of these recommendations at several institutions in the USA and other countries are described in a review by Li et al. [16]. Per AAO guidelines, patients with DR who obtain regular injections were advised to contact their ophthalmologists regarding treatment [15].

Vision Academy's Steering Committee, an international group of over 80 retina experts worldwide sponsored by Bayer, also provided specific guidelines regarding anti-vascular endothelial growth factor (VEGF) intravitreal injections for neovascular age-related macular degeneration (nAMD), diabetic macular edema (DME), and retinal vein occlusions (RVO) [17]. Anti-VEGF intravitreal injections are standard care for treatment of DME and proliferative diabetic retinopathy (PDR), the main mechanisms by which patients with DR lose vision.

General guidelines from the Vision Academy's Steering Committee included minimizing exposure risk by simplifying anti-VEGF regimen, prioritizing treatment for patients at risk of irreversible vision loss, and not changing treatment medications unless there was a clear lack of response. Pertaining to DR specifically, recommendations in the early part of the pandemic included deferral of anti-VEGF injections for established DME patients and re-evaluation after 4 months [17]. However, delayed treatment for severe non-proliferative diabetic retinopathy (NPDR) and active PDR was not recommended due to the risk of vision loss. For patients with a new diagnosis of DME, the committee recommended deferring treatment for 6 months. As the pandemic continued, updated recommendations by the Vision Academy's Steering Committee were published in 2021, which included treating patients with DME and significant vision loss, avoiding treatment postponement for more than 4-6 months, and re-evaluating patients within $2-3$ months [18].

\section{Real-World Impact on Practice Patterns}

The guidelines from ophthalmologists and retinal specialists as described, which aimed to minimize the risk of COVID19 exposure in patients with diabetic retinopathy, resulted in dramatic changes in clinical practice patterns. Patientrelated factors, particularly fear of COVID-19 exposure, also contributed significantly to loss of follow-up during the pandemic. In fact, in a survey at two tertiary eye care centers in the USA (Emory Eye Center in Atlanta, GA, and W.K. Kellogg Eye Center in Ann Arbor, MI), 47\% of patients with nAMD or DR who were scheduled to receive an injection between March 13 and May 6, 2020 and who responded to the survey were moderately to very concerned about vision loss from missed intravitreal injections during the pandemic. However, fear of exposure was associated with approximately fourfold increased odds of patient loss to follow-up [8]. A retrospective analysis of medical records in the UK of patients with nAMD, DME, and RVO who required injection revealed a $67 \%$ non-attendance rate during the first 4 weeks of lockdown due to the pandemic (March 23 to April 17, 2020) [19]. Overall, changes in guidelines and patient-related factors have significantly impacted clinic volume and also resulted in fewer intravitreal injections during the pandemic.

In a multicenter cross-sectional study across 17 institutions in the USA, billing data for vitreoretinal procedures from January 1, 2019 and May 21, 2020 were queried, yielding an aggregate of 526,536 procedures [20]. The average weekly intravitreal injections per institution for all indications were significantly lower in April 2020 compared to April 2019. The greatest decrease in intravitreal injections (38.6\% reduction) was noted in the week of April 6 to April 12. Differences in weekly intravitreal injection numbers were no longer significant by the end of May 2020 compared to the same time period in 2019. In a separate study focusing on three COVID-19 hot spots in the USA (New York, Boston, and Miami) from March 16 to May 8, 2020, the decline in intravitreal injections ranged from 30 to $64 \%$ [21]. 
Similar changes in practice patterns during the pandemic were also noted globally. A tertiary referral center in Italy, which deferred treatment for DME for up to 30-40 days, showed a $91.7 \%$ reduction in intravitreal injections for nAMD, DME, and RVO combined between March and May 2020 compared to the same time period in 2019 [22]. Specifically, 40 intravitreal injections were performed during the pandemic lockdown compared to 483 injections during the same time period in 2019. Interestingly, DME patients seemed to be disproportionally affected. Between March and May 2020 at the height of the pandemic, 75\% $(n=30 / 40)$ and $15 \%(n=6 / 40)$ of injections were administered for nAMD and DME, respectively [22]. In contrast, during the same period in 2019, 1 year prior, $46.4 \%(n=224 / 483)$ and $43.5 \%$ $(n=210 / 483)$ of intravitreal injections were administered for nAMD and DME, respectively [22]. In a different study, at the Policlinico Hospital in Milan, Italy, there was an $81 \%$ decrease in intravitreal injections during the national lockdown from March 8 to March 31, 2020 [23]. A retrospective analysis of ophthalmic practices in 39 academic centers in Italy between March 10 and May 9, 2020 revealed an almost $50 \%$ decline in intravitreal injections for all indications [24].

Other groups in different countries have also published variable loss of follow-up rates for intravitreal injection ranging from $50-70 \%$, likely a reflection of the different exposure risks and COVID-19-related public health policies in these regions [25, 26]. At the China Medical University First Hospital Department of Ophthalmology, a 70\% decrease in the number of intravitreal injections was noted between January 21, 2020 and June 1, 2020 when ambulatory clinic restrictions went into effect compared to the same time period in 2019 [25]. Moreover, 81.9\% of eyes had a delayed treatment of 4.5 months or longer. At the Shaare Zedek Medical Center in Israel, intravitreal injections decreased by approximately 50\% from March 15 to April 14, 2020 compared to projected injections calculated from reported injections during the same period for the prior 4 years [26]. This study included all indications for anti-VEGF injections.

Several studies have also investigated intravitreal injections during the reopening period following pandemicrelated lockdown. While the decrease in clinical volume was not as dramatic, the number of intravitreal injections administered did not experience full recovery. At the Bascom Palmer Eye Institute in the USA during the "new normal" of operations from June 18 to August 7, 2020, a 9.9\% decrease in intravitreal injections was noted compared to the corresponding period in 2019 [27]. Similarly, in France, there was a $11.5 \%$ decrease in observed intravitreal antiVEGF injections compared to expected injections (extrapolated from the prior two years) in the period following lockdown (May 11-June 7, 2020). [28] These studies suggested that missed intravitreal injections during lockdown were not compensated for during re-opening.
Clinic restrictions, closure of private clinics, resource shortages, travel restrictions, lack of public transport facilities, and patient concerns were all factors that contributed to the decrease in intravitreal injections [24, 25, 29]. Younger patients and those with worse visual acuity in the fellow eye were more likely to adhere to anti-VEGF treatment [23, 30]. As a result of the decreased clinic visits and intravitreal injections, several studies have investigated the impact of delayed care on functional and anatomic outcomes in patients with DR in recent months.

\section{Impact of Delayed Care on Visual Outcomes}

Retrospective analyses comparing best-corrected visual acuity (BCVA) before and after the onset of the COVID-19 pandemic have shown a variable impact of treatment delay on BCVA in patients with DR. Several institutions noted the negative impact of delayed treatment on visual acuity. In a retrospective analysis of patients receiving intravitreal injections at the Cole Eye Institute in the USA from March 14 to May 4, 2020, patients with DME and/or PDR whose appointments were delayed lost $3.48 \pm 1.95$ ETDRS letters compared to patients who completed their scheduled appointment (gained $2.71 \pm 1.75$ ETDRS letters, $p=0.0203$ ) [30]. The average delay in care for all patients with missed appointments was 5.34 weeks. In a retrospective analysis of patients receiving anti-VEGF injections at the University of Minnesota Retina Clinic and the Retina Center in Minneapolis between March 28, 2020 and September 30, 2020 , delayed treatment for patients with nAMD, DME, or RVO resulted in worse BCVA at follow-up. Within the DME subgroup, patients whose injections were delayed trended towards a decline in vision from logMAR 0.544 (Snellen 20/70) pre-lockdown to logMAR 0.722 (Snellen 20/105) on follow-up $(p=0.06)$. In contrast, patients whose injections were not delayed did not experience a statistically significant decline in vision $(p=0.40)$ [31]. Delayed anti-VEGF treatment in patients with DME also resulted in an increase in mean central subfield thickness from 341 to $447 \mu \mathrm{m}$ $(p=0.007)$.

Similar changes in BCVA due to delayed treatment were seen in other countries. At the China Medical University First Hospital Department of Ophthalmology, the average treatment interruption length was $5.3 \pm 0.8$ months and the BCVA decreased from logMAR $0.57 \pm 0.23$ (Snellen 20/74) before treatment interruption to $\operatorname{logMAR} 0.98 \pm 0.41$ (Snellen 20/191) on return visit for patients with DME, nAMD and RVO [25]. Specifically, $66.7 \%$ of patients with DME lost 3 or more BCVA lines on the return visit. On Pearson's correlation analysis and multivariate analysis, longer treatment interruption was correlated with worse BCVA. At the Jordan University of Science and Technology, anti-VEGF treatment 
delays resulted in worse visual acuity in all patients requiring intravitreal injections, including patients with DME [32]. The average delay was $6.2 \pm 1.4$ weeks during lockdown [32]. A retrospective observational study at the Jordan University Hospital from April 20 to July 1, 2020, showed that delayed intravitreal injections of more than 2 months during the COVID-19 lockdown, as well as the prior need for 3 or more injections, were poor prognostic factors for visual acuity in patients with DME at the return visit following treatment delay [33]. The average delay in injections in this study was $60.97( \pm 24.35)$ days.

However, several other groups did not note a change in functional outcomes following treatment delays. The Tanta University Hospitals Ophthalmology Department in Egypt did not note any significant change in BCVA following delayed treatment, using guidelines similar to the 2021 updated Vision Academy recommendations [34]. The Aravind Eye Hospital in India also did not note any change in BCVA before and after delayed treatment of DME despite a longer treatment delay of $19.1 \pm 10.6$ weeks [29].

These studies were all limited by their retrospective nature, which are prone to selection biases. This could explain the conflicting conclusions and varying degree of impact on BCVA due to deferred anti-VEGF treatment. To fully understand the impact of COVID-19 on DR-related morbidity, population-based studies are needed.

\section{Conclusion}

COVID-19 has caused unprecedented upheavals around the world and has dramatically impacted clinical care. Specifically for DR, COVID-19 has led to a decrease in clinic visits and administration of anti-VEGF injections, which in turn likely adversely affected patients' vision outcome, though the data is not conclusive. More rigorous studies on a population level are needed to fully evaluate the impact of COVID-19 on DR-related outcomes and whether the associated negative outcomes are permanent. Moreover, a multicenter analysis of pandemic-associated changes in retinal procedures and surgeries demonstrated a decrease in the number of laser procedures, vitrectomies for retinal detachment repair, and vitrectomies for other indications [20]. Further work will be needed to determine the impact of the pandemic on PDR-specific laser procedures and vitrectomies.

The COVID-19 pandemic has also highlighted the need for telemedicine in diabetic retinopathy screening and monitoring. Patient self-screening, which has been shown to be effective using the near card [35] and Alleye program [36], may help optimize prioritization protocols for clinic visit and intravitreal injections. Moreover, telescreening using fundus photography and artificial intelligence-based classification of diabetic retinopathy have demonstrated high specificity and sensitivity in diagnosing diabetic retinopathy [37-39]. Furthermore, home-based monitoring of patients with DME with portable OCT systems will likely be beneficial [40]. Although numerous implementation challenges, such as infrastructure costs and changes in workflow in nonophthalmic settings, remain, large-scale tele-ophthalmology programs, when combined with artificial intelligence technologies and at-home monitoring devices, will be beneficial to patients with diabetic retinopathy by improving access to care, particularly in the context of the COVID-19 pandemic.

\section{Declarations}

Conflict of interest The authors declare no competing interests.

\section{References}

1. WHO COVID-19 Dashboard. Geneva: World Health Organization. Available online: https://covid19.who.int/ (last cited: 4/9/2021)

2. Liu PP, Blet A, Smyth D, Li H. The Science Underlying COVID19: Implications for the Cardiovascular System. Circulation. 2020;142(1):68-78.

3. Lee R, Wong TY, Sabanayagam C. Epidemiology of diabetic retinopathy, diabetic macular edema and related vision loss. Eye Vis (Lond). 2015;2:17.

4. Bourne RR, Stevens GA, White RA, Smith JL, Flaxman SR, Price H, et al. Causes of vision loss worldwide, 1990-2010: a systematic analysis. Lancet Glob Health. 2013;1(6):e339-49.

5. Schoenfeld ER, Greene JM, Wu SY, Leske MC. Patterns of adherence to diabetes vision care guidelines: baseline findings from the Diabetic Retinopathy Awareness Program. Ophthalmology. 2001;108(3):563-71.

6. Preliminary report on effects of photocoagulation therapy. The Diabetic Retinopathy Study Research Group. Am J Ophthalmol. 1976;81(4):383-96.

7. Kirkizlar E, Serban N, Sisson JA, Swann JL, Barnes CS, Williams MD. Evaluation of telemedicine for screening of diabetic retinopathy in the Veterans Health Administration. Ophthalmology. 2013;120(12):2604-10

8. Lindeke-Myers A, Zhao PYC, Meyer BI, Liu EA, Levine DA, Bennett OM, et al. Patient perceptions of SARS-CoV-2 exposure risk and association with continuity of ophthalmic care. JAMA Ophthalmol. 2021.

9. Centers for Disease Control and Prevention. Infection Control Guidance for Healthcare Professionals about Coronavirus (COVID-19)2020 April 9, 2021. Available from: https://www. cdc.gov/coronavirus/2019-ncov/hcp/infection-control.html.

10. Strata Decision Technology National Patient and Procedure Volume Tracker. Published May 11, 2020 April 10, 2021. Available from: https://www.stratadecision.com/wp-content/uploads/2020/ 05/National-Patient-and-Procedure-Volume-Tracker-and-Report May2020.pdf.

11. Xu D, Starr MR, Boucher N, Chiang A, Yonekawa Y, Klufas MA, et al. Real-world vitreoretinal practice patterns during the 2020 COVID-19 pandemic: a nationwide, aggregated health record analysis. Curr Opin Ophthalmol. 2020;31(5):427-34. 
12. Shih KC, Kwong ASK, Wang JHL, Wong JKW, Ko WWK, Lai JSM, et al. Diabetic retinopathy screening during the coronavirus disease 2019 pandemic. Eye (Lond). 2020;34(7):1246-7.

13. Berkenstock MK, Liberman P, McDonnell PJ, Chaon BC. Changes in patient visits and diagnoses in a large academic center during the COVID-19 pandemic. BMC Ophthalmol. 2021;21(1):139.

14. Prevention CfDCa. COVID-19: People at Increased Risk2021 April 9, 2021 Available from: https://www.cdc.gov/coronavirus/ 2019-ncov/need-extra-precautions/index.html.

15. Ophthalmology AAo. Eye Care During the Coronavirus Pandemic (COVID-19)2020 April 9, 2021.

16. Olivia Li JP, Shantha J, Wong TY, Wong EY, Mehta J, Lin H, et al. Preparedness among ophthalmologists: during and beyond the COVID-19 pandemic. Ophthalmology. 2020;127(5):569-72.

17. Korobelnik JF, Loewenstein A, Eldem B, Joussen AM, Koh A, Lambrou GN, et al. Guidance for anti-VEGF intravitreal injections during the COVID-19 pandemic. Graefes Arch Clin Exp Ophthalmol. 2020;258(6):1149-56.

18. Korobelnik JF, Loewenstein A, Eldem B, Joussen AM, Koh A, Lambrou GN, et al. Anti-VEGF intravitreal injections in the era of COVID-19: responding to different levels of epidemic pressure. Graefes Arch Clin Exp Ophthalmol. 2021;259(3):567-74.

19. Stone LG, Devenport A, Stratton IM, Talks JS. Macula service evaluation and assessing priorities for anti-VEGF treatment in the light of COVID-19. Graefes Arch Clin Exp Ophthalmol. 2020;258(12):2639-45.

20. Breazzano MP, Nair AA, Arevalo JF, Barakat MR, Berrocal AM, Chang JS, et al. Frequency of urgent or emergent vitreoretinal surgical procedures in the United States During the COVID-19 pandemic. JAMA Ophthalmology. 2021.

21. El Hamichi S, Gold A, Heier J, Kiss S, Murray TG. Impact of the COVID-19 pandemic on essential vitreoretinal care with three epicenters in the United States. Clin Ophthalmol. 2020;14:2593-8.

22. Carnevali A, Giannaccare G, Gatti V, Scuteri G, Randazzo G, Scorcia V. Intravitreal injections during COVID-19 outbreak: real-world experience from an Italian tertiary referral center. Eur J Ophthalmol. 2021;31(1):10-2.

23. Viola F, Milella P, Pozzo Giuffrida F, Ganci S, Invernizzi A. IMPACT OF CORONAVIRUS DISEASE PANDEMIC ON INTRAVITREAL INJECTIONS TREATMENT FOR MACULAR DISEASES: Report From a Referral Hospital in Milan. Retina. 2021;41(4):701-5.

24. dell'Omo R, Filippelli M, Virgili G, Bandello F, Querques G, Lanzetta P, et al. Effect of COVID-19-related lockdown on ophthalmic practice in Italy: a report from 39 institutional centers. Eur J Ophthalmol. 2021:11206721211002442.

25. Yang KB, Feng H, Zhang H. Effects of the COVID-19 pandemic on anti-vascular endothelial growth factor treatment in China. Front Med (Lausanne). 2020;7:576275.

26. Wasser LM, Weill Y, Brosh K, Magal I, Potter M, Strassman I, et al. The impact of COVID-19 on intravitreal injection compliance. SN Compr Clin Med. 2020:1-4.

27. Ashkenazy N, Goduni L, Smiddy WE. Short-term effects of COVID-19-related deferral of intravitreal injection visits. Clin Ophthalmol. 2021;15:413-7.

28. Billioti de Gage S, Drouin J, Desplas D, Cuenot F, Dray-Spira R, Weill A, et al. Intravitreal anti-vascular endothelial growth factor use in France during the coronavirus disease 2019 pandemic. JAMA Ophthalmol. 2021;139(2):240-2.
29. Sindal MD, Chhabra K, Khanna V. Profile of patients receiving intravitreal anti-vascular endothelial growth factor injections during COVID-19-related lockdown. Indian J Ophthalmol. 2021;69(3):730-3.

30. Song W, Singh RP, Rachitskaya AV. The effect of delay in care among patients requiring intravitreal injections. Ophthalmol Retina. 2021.

31. Naravane AV, Mundae R, Zhou Y, Santilli C, van Kuijk F, Nazari $\mathrm{H}$, et al. Short term visual and structural outcomes of antivascular endothelial growth factor (anti-VEGF) treatment delay during the first COVID-19 wave: a pilot study. PLoS One. 2021;16(2):e0247161.

32. Saleh OA, Jammal H, Alqudah N, Alqudah A, Abu-Yaghi N. Clinical experience in the administration of intravitreal injection therapy at a tertiary university hospital in jordan during the COVID-19 lockdown. Clin Ophthalmol. 2020;14:2473-80.

33. Elfalah M, AlRyalat SA, Toro MD, Rejdak R, Zweifel S, Nazzal R, et al. Delayed Intravitreal anti-VEGF therapy for patients during the COVID-19 lockdown: an ethical endeavor. Clin Ophthalmol. 2021;15:661-9.

34. Moussa M, Elshorbagy MS, Emarah A, Gaber R, Moussa OM, El-Bradey M, et al. Intravitreal injections of anti-VEGF agents during COVID-19 pandemic: clinical audit from Tanta University Hospital. Int Ophthalmol. 2021:1-7.

35. Tiraset N, Poonyathalang A, Padungkiatsagul T, Deeyai M, Vichitkunakorn P, Vanikieti K. Comparison of visual acuity measurement using three methods: standard ETDRS chart, near chart and a smartphone-based eye chart application. Clin Ophthalmol. 2021;15:859-69.

36. Teo KYC, Bachmann LM, Sim D, Lee SY, Tan A, Wong TY, et al. Patterns and characteristics of a clinical implementation of a self-monitoring program for retina diseases during the COVID-19 Pandemic. Ophthalmol Retina. 2021.

37. Bawankar P, Shanbhag N, K SS, Dhawan B, Palsule A, Kumar $\mathrm{D}$, et al. Sensitivity and specificity of automated analysis of single-field non-mydriatic fundus photographs by Bosch DR Algorithm-Comparison with mydriatic fundus photography (ETDRS) for screening in undiagnosed diabetic retinopathy. PLoS One. 2017;12(12):e0189854.

38. Malerbi FK, Andrade RE, Morales PH, Stuchi JA, Lencione D, de Paulo JV, et al. Diabetic retinopathy screening using artificial intelligence and handheld smartphone-based retinal camera. J Diabetes Sci Technol. 2021:1932296820985567.

39. Li JO, Liu H, Ting DSJ, Jeon S, Chan RVP, Kim JE, et al. Digital technology, tele-medicine and artificial intelligence in ophthalmology: a global perspective. Prog Retin Eye Res. 2021;82:100900.

40. Kim S, Crose M, Eldridge WJ, Cox B, Brown WJ, Wax A. Design and implementation of a low-cost, portable OCT system. Biomed Opt Express. 2018;9(3):1232-43.

Publisher's Note Springer Nature remains neutral with regard to jurisdictional claims in published maps and institutional affiliations. 